Reseña 


\title{
Enunciación
}

\section{Repensar identidades y literacidades, reimaginar pedagogías posibles: aportes de miradas desde la transculturalidad y el cosmopolitismo}

\author{
Reseña de Literacy Lives in Transcultural Times
}

\author{
María Sol Iparraguirre*
}

Literacy Lives in Transcultural Times (2017) es una compilación de estudios en los que se analizan formas de construcción identitaria, a partir de prácticas de literacidad en escenarios socioeducativos actuales, marcados por la transculturalidad, el cosmopolitismo y la descentralización (de medios, de lenguajes, de normas, de culturas). El libro fue editado por Rahat Zaidi y Jennifer Rowsell, ambas de extensa trayectoria en investigaciones sobre diversidad lingüística y cultural, pedagogías multilingües, y prácticas de literacidad y entornos digitales, y prologado por Marjorie Faulstich Orellana. La introducción, escrita por las editoras, expone los fundamentos teóricos rectores del libro en su conjunto y los doce capítulos que le siguen presentan trabajos de investigación que comparten la problematización de categorías en gran medida naturalizadas, a partir de estudios de caso.

En el capítulo introductorio, las editoras parten de las nociones de transculturalidad y cosmopolitismo como herramientas teóricas vertebradoras para abordar cuestiones de diversidad lingüística y cultural, semiosis, multimodalidad e identidad, como forma de tender puentes entre estudiantes y educadores que no se parecen entre sí. De acuerdo con este enfoque, la centralidad de la transculturalidad en las sociedades contemporáneas es resultado de los cambios culturales que devienen (en el pensar, en el sentir, en el ser) cuando las personas se mueven espacialmente, sea de forma temporaria o permanente, y de manera física o digital. Los estudios que conforman el libro abarcan distintos modos en que las prácticas lingüísticas, discursivas y multimodales también se mueven, transforman y resignifican, creando nuevos contextos o recreando los conocidos, y se constituyen en instancias tanto para la proyección de identidades, como para su (re)definición. En esta línea, el cosmopolitismo se redefine como el proceso de construcción de identidad en un ser y hacer situado y dinámico, que se proyecta a un mundo policéntrico (Blommaert y Backus, 2011). Los editores destacan la necesidad de deconstruir y descentralizar la manera de investigar y enseñar, como forma de romper con la visión sobre el otro basada en el conflicto o en su idealización, y de aportar

* Universidad Nacional de Río Negro. Centro de Estudios del Lenguaje, la Literatura, su Aprendizaje y su Enseñanza (CELLAE). Río Negro, Argentina. msiparraguirre@unrn.edu.ar

Cómo citar: Iparraguirre, M. S. (2021). [Reseña] Repensar identidades y literacidades, reimaginar pedagogías posibles: aportes de miradas desde la transculturalidad y el cosmopolitismo. Enunciación, 26, 171-176. DOI: https://doi.org/10.14483/22486798.17127 
a formas más democráticas, tanto en la educación como en la participación ciudadana, sin negar diferencias y fronteras, sino incorporándolas (en el sentido propuesto por el embodyment, cfr. Varela, Thompson y Rosch, 1991), para el mutuo conocimiento, reconocimiento y comprensión.

Los capítulos que siguen a la introducción son agrupados en tres secciones, según espacios o panoramas desde los cuales se analizan la transculturalidad y el cosmopolitismo en relación con distintas prácticas lingüísticas y de literacidad, conforme la propuesta de Appadurai ${ }^{1}$ : Ethnoscapes, Technoscapes e Ideoscapes. Estos espacios constituyen principios organizadores para analizar el modo en que las identidades confluyen y median localmente, a la vez que se proyectan globalmente. A continuación se describirá brevemente cada sección y aspectos a resaltar de los capítulos que las integran, con el propósito de presentar una visión general del libro. Solo será abordado con más detalle el primer capítulo de Ethnoscapes, por considerarlo representativo del planteamiento teórico y metodológico de los editores, y por presentar una problemática vinculada a la historia de colonización, compartida por todos los pueblos americanos.

La primera sección, Ethnoscapes, refiere al paisaje lingüístico y cultural cambiante como producto del movimiento permanente o transitorio de personas y a los desbalances de poder que se producen en comunidades con historias de subyugación o marginación, pasadas o presentes. En el primer capítulo ("Complicating Literacies. Settler ways of Being with Story(ies) on Wabanaki Lands"), Pam Whitty se propone dislocar las literacidades, las construcciones históricas y el territorio marginalizados y colonizados (y a sí misma), a partir de conjugar relatos y teorizaciones, tanto dominantes como subyugados. A lo largo del texto, devela el grado de imbricación que la historia blanca, inglesa y protestante tiene en tierras del país de los

El antropólogo Arjun Appadurai es considerado uno de los pensadores más influyentes sobre globalización (Coleman y Sajed, 2013).
Wabanaki², en las instituciones y en ella misma, en cuanto académica canadiense blanca y habitante de dicho territorio. En consistente primera persona, la historia familiar, personal y profesional se entreteje con la historia de la colonización de ese espacio. El análisis, realizado desde el cuestionamiento de la propia identidad, encarna el cuestionamiento a pedagogías que continúan sin incorporar las epistemologías, las ontologías y los valores de los pueblos indígenas para la construcción de prácticas reflexivas superadoras de conceptualizaciones que, aún hoy, trasuntan un romanticismo dicotómico. La autora basa su estudio en las tensiones que se suscitan en territorio Wabanaki producto de las relaciones de poder vinculadas a la(s) historia(s) de colonización. Para ello retoma la descripción de la educadora blanca de origen europeo de Susan Dion (posteriormente ampliada por Marc Higgins) como la identidad de la perfecta extraña, imagen de las tensiones entre el colonizador perpetuante y el colonizador aliado, de la cual ella misma constituye una representante simbólica. Desde una mirada crítica sobre la historia territorial y personal, la autora señala que estas construcciones identitarias se sostienen en mecanismos cuya deconstrucción requiere una pedagogía descolonizadora basada en la solidaridad (siguiendo la propuesta de Rubén Gaztambide-Fernández), a fin de reconfigurar los encuentros interpersonales reordenando las jerarquías simbólicas que producen desencuentros. Whitty sostiene que la descolonización de las formas de enseñar y producir conocimiento requiere descentralizar la mirada occidental-europea en dirección a indigenizar el currículum y las aulas, como modo de forjar relaciones equitativas. Así, la transculturalidad y el cosmopolitismo se ponen en juego en un cambio de mirada que se mueve desde la parte hacia el todo, desde el ámbito más íntimo, hacia la construcción social, curricular y territorial. El análisis propuesto por la autora, si bien aborda el contexto Wabanaki en Canadá,

\footnotetext{
2 El "país de los Wabanaki" abarca zonas del este de Canadá y del noreste de Estados Unidos, y agrupa población de cinco pueblos indígenas de la región.
} 
bien podría pensarse para otros países americanos con equivalentes historias de colonización y subyugación de pueblos originarios, lo cual resulta en un aporte esencial para educadores/investigadores en estos contextos.

En esta misma sección, Brian Street ("International Struggles over 'Low Literacy' versus the Alternative 'Social Practices' Approach") revisa distintos programas que buscan superar las visiones deficitarias aún presentes en las evaluaciones internacionales sobre alfabetización, basadas en el testeo de habilidades, en contraste con su concepción como práctica social. Este enfoque permite pensar las trayectorias de alfabetización de los niños en términos de literacidad social, una mirada sensible al contexto y las necesidades locales que descentraliza transculturalmente la alfabetización. En línea con este planteamiento, Margaret Early y Maureen Kendrick ("Multiliteracies Reconsidered. A 'Pedagogy of Multiliteracies' in the Context of Inquiry-Based Approaches") analizan la intersección entre los postulados centrales de la pedagogía de la multiliteracidad (New London Group), los recursos multimodales para la construcción textual y la diversidad lingüística. Las autoras destacan el potencial que tiene una propuesta didáctica situada y con énfasis equivalente a la diversidad lingüística y al diseño pedagógico para promover el empoderamiento de los estudiantes, y concluyen que este tipo de diseños reposicionan roles y responsabilidades de educandos y educadores, abriendo la posibilidad para transformar la propia identidad a través del compromiso y la participación. De forma similar, Burcu Yaman Ntelioglou ("Examining the Relational Space of the Self and Other in the Language-Drama Classroom. Transcultural Multiliteracies, Situated Practice and the Cosmopolitan Imagination") plantea la necesidad de incorporar el interjuego de identidades plurales para pensar el currículum como práctica social y compuesto por sujetos y subjetividades.

La segunda sección del libro, Technoscapes, se centra en el paisaje de límites y fronteras difusos que devienen de la velocidad y el acortamiento de distancias (físicas y simbólicas) que producen las nuevas tecnologías de la información y la comunicación (TIC). Es la sección más extensa del libro y muestra la complejización que deriva de un mundo "crecientemente inalámbrico y digitalmente conectado" (Darvin y Norton, p. 89, traducción propia). En contextos de migración y transculturalidad, las personas no dependen ya de la localización geográfica ni de la congregación física para mitigar el aislamiento y la segregación (o incluso la discriminación), sino de la conexión individual en tiempo real a través de redes sociales y sitios de internet. En tanto flujos culturales mediados tecnológicamente, según Appadurai, estos espacios tecnológicos permiten del mismo modo la reinvención identitaria con proyección global, como el refuerzo o mantenimiento de una identidad vinculada a una raigambre local, siendo estas tal vez las formas más tangibles del cosmopolitismo. Por ejemplo, Karen Wohlwend y Carmen Medina ("Monster High: Converging Imaginaries of Girlhood in Tweens' Digital Doll Play") estudian en qué medida niñas canadienses reproducen o deconstruyen las representaciones de género normalizadoras (particularmente sobre el cuerpo), al participar en juegos de muñecas en línea.

Por su parte, Amy Stornaiuolo y Jin Kyeong Jung ("Public Engagement and Digital Authoring. Korean Adolescents Write for/as Action") analizan la trayectoria de una estudiante surcoreana a partir de prácticas de escritura digitales en el marco del programa Write4Change. Las autoras plantean la necesidad de formar a estudiantes como ciudadanos de un mundo globalmente conectado que puedan utilizar las herramientas digitales en forma efectiva y ética, lo que desafía a los educadores a promover situaciones de aprendizaje que potencien el valor transcultural de estas herramientas.

Sin embargo, Ron Darvin y Bonny Norton ("Investing in New Literacies for a Cosmopolitan Future") advierten que estas nuevas formas de interacción pueden profundizar el aislamiento y la fragmentación, y conllevan el riesgo de perder de vista que se encuentran igualmente constreñidas 
por relaciones de poder, aspectos que interpelan a analizar críticamente las implicaciones pedagógicas de la transculturalidad y el cosmopolitismo, tanto en sus potencialidades como en sus contradicciones e inequidades. Estos autores basan sus reflexiones en dos estudios de caso en los que participaron adolescentes de contextos geográficos y sociales diametralmente diferentes (un inmigrante sirio en Canadá y una joven ugandesa), mostrando que experiencias similares con artefactos y prácticas digitales no logran anular concepciones -ni promover un posicionamiento como agentes cosmopolitas- cuya raíz se encuentra en las inequidades de base.

En línea con lo previo, Diane Collier ("Rescripting Classed Lives and Imagining Audiences as Online Cosmopolitan Practice") y Michelle Honeyford ("Artifacts as Catalysts for Reimagining Transcultural Literacy Pedagogies ") estudian la potencialidad de los artefactos (dispositivos, soportes) y las propuestas pedagógicas para renovar el currículum y fomentar movimientos transculturales que trasciendan condicionantes de base ligados a los grupos sociales de origen, crecientemente diversos y complejos.

La tercera sección, Ideoscapes, refiere a las representaciones en torno a conceptos rectores de vida ciudadana que circulan a través de textos y redes sociales, y se funda en la amplia, veloz e incesante circulación de opiniones e ideologías por medio de las TIC y como consecuencia de migraciones. Esto conlleva una alta exposición, definición y redefinición de las subjetividades, y también la creación de comunidades a partir de la identificación ideológica. Los nuevos escenarios de atomización ideológico-subjetiva y de redefinición de la materialidad humana, en relación con los artefactos electrónicos, desafían a educadores e investigadores.

Candace Kuby ("Poststructural and Posthuman Theories as Literacy Research Methodologies. Tensions and Possibilities") se sitúa dentro del paradigma posestructuralista y poshumanista y propone un cambio de mirada sobre las prácticas de literacidad basado en las posteorías, que incorpore un giro material (como ya sucedió con el giro lingüístico). La autora sostiene que el cambio de paradigma impone problematizar y modificar todos los aspectos de la investigación para poder captar los componentes (humanos y no humanos) en el modo de crear, ser y hacer literacidad.

Por otro lado, Anne Crampton, Cynthia Lewis y Jessica Dockter Tierney ("Proper Distance and the Hope of Cosmopolitanism in a Classroom Discussion about Race") estudian interacciones entre docentes y estudiantes cuyas historias y lealtades se ubican en múltiples locaciones y comunidades a partir del concepto de translocalización, para analizar cómo los efectos de la relocalización, que enfrenta a los sujetos con otras formas de pensar, sentir y hacer, pueden ser tanto el refuerzo como la negación o el cuestionamiento de las propias pautas idiosincráticas y de los rasgos más encarnados de su identidad (clase social, raza, género). Las autoras adoptan una mirada amplia sobre la migración que, además del traslado de un país a otro, concierne las cambiantes distancias entre personas, cuerpos, objetos y signos, que implican movilidades en distintas escalas de tiempo y espacio.

La sección cierra con el capítulo de Patriann Smith, S. Joel Warrican y Gwendolyn Williams ("Towards Transculturalism in Tackling Diversity for Literacy Teacher Education"), quienes trabajan la diferencia como fundamento de espacios transculturales, corriendo el foco de la identificación de diferencias en el interior de grupos de estudiantes diversos, para repensar la diversidad como un constructo existente entre estudiantes y docentes.

Tal como muestra el recorrido previo, este volumen concentra una importante cantidad y variedad de estudios. Cada uno de ellos busca desnaturalizar algún aspecto de la construcción de la mirada sobre el otro, a partir de mostrar la diversidad como constitutiva de las sociedades y de las prácticas de literacidad actuales. El eje que vertebra y da unidad al volumen habilita tal concentración de estudios; no obstante, la brevedad de los capítulos conlleva condensaciones en el desarrollo conceptual o metodológico, que en 
ocasiones dificulta al lector situarse acabadamente en las propuestas. Así sucede, por ejemplo, con la noción de identidad, por momentos difícil de aprehender, así como con aspectos procedimentales de los estudios de caso. Asimismo, la problematización de categorías naturalizadas se habría beneficiado de una mayor atención a la centralidad de la escritura en los procesos de literacidad estudiados, particularmente en torno a las tecnologías digitales, en relación justamente con la diversidad sociocultural planteada.

El objetivo último de las editoras, encontrar estrategias de transformación educativa en sociedades que se mueven en la tensión entre verdaderamente incorporar la diversidad y discriminar (de formas tangibles o no) en base al desconocimiento e incomprensión, resulta tan amplio y global, como urgente y actual. Las propuestas de este volumen se encuentran atravesadas por un dilema de especial relevancia en este contexto de voraz y vertiginosa virtualización de la vida académica, laboral y personal: o bien la educación y la política trabajarán para zanjar la desigualdad, o bien la profundizarán. Cada estudio aporta a la superación de dicotomías que encuentran su raíz en la ya clásica contraposición sujeto "normal"/sujeto estigmatizado, con sus correspondencias sobre la construcción identitaria (Goffman, 1963), y buscan abordar las desigualdades desde propuestas de acción político-educativas.

Literacy Lives in Transcultural Times, tal como sostiene Orellana, desafía al lector a "animarse" a pensar y diseñar otro mundo posible (y necesario), cuyas claves serán la transculturalidad, el cosmopolitismo, la interconectividad y la empatía, para así derribar definitivamente las fronteras con el otro. A pesar de tratarse de un libro de moderada extensión, la revisión conceptual y la minuciosa articulación entre investigación y diseño pedagógico que presentan la mayoría de los estudios contribuyen a problematizar y repensar categorías y procedimientos naturalizados en la investigación cualitativa, aspectos que lo convierten en una lectura necesaria para investigadores en estas temáticas. A lo largo de los capítulos se interpela al lector a convertirse en parte del cambio propuesto; en este sentido, investigadores y educadores en prácticas de literacidad difícilmente puedan continuar realizando sus tareas del mismo modo, luego de leer este libro.

\section{Referencias bibliográficas}

Blommaert, J. y Backus, A. (2011). Repertoires revisited: 'Knowing language' in superdiversity. Working Papers in Urban Language \& Literacies, 67. Londres: King's College. Recuperado de http://www.kcl. ac.uk/sspp/departments/education/research/ldc/ publications/workingpapers/67.pdf

Coleman, W. D. y Sajed, A. (2013). Fifty key thinkers on globalization. Abingdon/Nueva York: Routledge. DOI: https://doi.org/10.4324/9780203080054

Goffman, E. (1963). Stigma: Notes on the management of spoiled identity. Nueva York: Touchstone.

Varela, F., Thompson, E. y Rosch, E. (1991). The embodied mind: Cognitive science and human experience. Cambridge: MIT Press. DOI: https://doi. org/10.7551/mitpress/6730.001.0001 


\section{Ficha técnica}

Título del libro: Literacy Lives in Transcultural Times

Editores: Rahat Zaidi (Werklund School of Education, University of Calgary, Canadá) y Jennifer Rowsell

(Centre for Multiliteracies in the Faculty of Education, Brock University, Canadá)

Colección: Expanding Literacies in Education

Editorial: Routledge

Idioma: Inglés

(C) 2017 Taylor \& Francis

ISBN: 978-1-138-22515-2 (hbk)

ISBN: 978-1-138-22516-9 (pbk)

ISBN: 978-1-315-40086-0 (ebk) 